\title{
Concepciones sobre los sistemas de adquisición de datos en el trabajo de laboratorio en Física
}

\author{
Conceptions about data acquisition systems in Physics laboratory work
}

\section{Concepções sobre aquisiç̧ão automática de dados no traballho de laboratório em Física}

\author{
Mónica Eliana Cardona-Zapata' \\ Daniel Pabón-Rúa ${ }^{2}$ (i) \\ Sonia Yaneth López-Ríos ${ }^{3}$
}

Recibido: mayo de 2020

Aceptado: octubre de 2020

Para citar este artículo: Cardona-Zapata, M.E., Pabón-Rúa, D., López-Ríos, S.Y. (2021). Concepciones sobre los sistemas de adquisición de datos en el trabajo de laboratorio en Física. Revista Científica, 40(1), 74-88.

https://doi.org/10.14483/23448350.16295

\section{Resumen}

Se ha reportado en la literatura especializada que en los trabajos prácticos de laboratorio en Física se presenta una clara escisión entre teoría y práctica que da cuenta de una visión distorsionada de la construcción del conocimiento científico. Además, dicha visión ha hecho que se vea desfavorecido el desarrollo de procesos cognitivos de orden superior de los sujetos, por limitar este espacio de aprendizaje al seguimiento de guías pautadas con objetivos preestablecidos. En este sentido, se construyó una propuesta teórico-metodológica fundamentada en la teoría de los campos conceptuales de Gerard Vergnaud, en la que se articulan los trabajos prácticos de laboratorio y el uso de tecnologías como los sistemas de adquisición de datos (SAD) para abordar situaciones que favorezcan la conceptualización y, por tanto, los procesos de representación que adquieren gran importancia al considerar el carácter abstracto de la Física. La propuesta se puso en marcha con un grupo de futuros profesores del área de Ciencias Naturales de un programa de Licenciatura de una universidad pública, con quienes se llevó a cabo un estudio de caso desde una perspectiva cualitativa. A partir de los resultados se puede afirmar que los SAD permiten establecer relaciones entre variables, reducen la incertidumbre en la toma de datos y desempeñan un papel importante en el proceso de representación de los fenómenos físicos. Palabras clave: conceptualización, enseñanza de la física, formación de profesores, sistemas de adquisición de datos, trabajo de laboratorio en Física.

\begin{abstract}
It has been reported in the literature that in the practical laboratory work in physics there is a clear split between theory and practice that accounts for a distorted view of the construction of scientific
\end{abstract}

1. Universidad de Antioquia. Medellín, Colombia. meliana.cardona@udea.edu.co

2. Universidad de Antioquia. Medellín, Colombia. jhon.pabon@udea.edu.co

3. Universidad de Antioquia. Medellín, Colombia. sonia.lopez@udea.edu.co 
knowledge. In addition, this vision has made the development of higher-order cognitive processes of the subjects to be disadvantaged by limiting this learning space to following guidelines conducted with pre-established objectives. In this sense, a theoretical-methodological proposal was built based on Gerard Vergnaud's Theory of Conceptual Fields, in which practical laboratory work and the use of technologies such as data acquisition systems (DAS) are articulated to address situations that favor conceptualization; therefore, favoring the representation processes that acquire great importance when considering the abstract nature of physics. The proposal was implemented with a group of future teachers in the area of natural sciences from a Bachelor's program at an official university, with whom a case study was carried out from a qualitative perspective. From the results, it can be affirmed that SAD allow relationships between variables to be established, reduce uncertainty in data collection, and play an important role in the process of representing physical phenomena.

Keywords: conceptualization, physics teaching, teacher training, data acquisition system, physics laboratory.

\section{Resumo}

Na literatura amostra que, no trabalho prático de laboratório em física, existe uma divisão entre teoria e prática que da conta de uma visão distorcida da construção do conhecimento científico. Além disso, essa visão diminui o desenvolvimento de processos cognitivos de ordem superior dos sujeitos, limitando esse espaço de aprendizado a seguir diretrizes orientadas com objetivos pré-estabelecidos. Nesse sentido, foi construida uma proposta teórico-metodológica baseada na teoria dos campos conceituais de Gerard Vergnaud, na qual o trabalho prático de laboratório e o uso de tecnologias como o data acquisition systems (SAD) são articulados para abordar situações que favorecem a conceituação e, por tanto, processos de representação que adquirem grande importância ao considerar a natureza abstrata da física. A proposta foi implementada com um grupo de futuros professores da área de ciências naturais de um bacharelado da uma universidade pública, e foi realizado um estudo de caso desde uma perspectiva qualitativa. A partir dos resultados, pode-se afirmar que o SAD permite estabelecer relações entre variáveis, reduzir a incerteza na coleta de dados e desempenhar um papel relevante no processo de representação dos fenômenos físicos.

Palavras-chaves: conceituação, ensino de física, formação de professores, aquisição automática de dados, trabalho de laboratório em física.

\section{Introducción}

Este texto forma parte de una investigación en la que se buscó identificar la contribución de los sistemas de adquisición de datos (SAD) a los procesos de conceptualización de un grupo de profesores en formación del área de Ciencias Naturales al momento de enfrentar diversas situaciones en el marco del trabajo práctico en el laboratorio de Física. Esto con el fin de tener claros los elementos que permitan construir ambientes de aprendizaje en los cuales los sujetos tengan la oportunidad de desarrollar habilidades de pensamiento de orden superior ${ }^{4}$, adaptarse cognitivamente a las circunstancias y apropiarse del acervo cultural que hasta ahora ha construido la humanidad, particularmente del conocimiento científico.

La propuesta se fundamentó en la teoría de los campos conceptuales de Vergnaud (1990), por su énfasis en la conceptualización a partir de situaciones que ponen en juego diversos componentes de la estructura cognitiva de los estudiantes. En concordancia con dicha propuesta, se analizaron las concepciones de los profesores en formación en relación con la función que le otorgaron a los SAD como apoyo en la recolección de datos, la medición de magnitudes y los procesos de representación de los fenómenos físicos.

\section{Uso de SAD para la enseñanza de la Física}

Los SAD han sido ampliamente utilizados en el área de Ciencias como un recurso que favorece

\footnotetext{
4. Según Zohar (2006), se entienden como: argumentar, comparar, analizar, identificar suposiciones subyacentes en controversias y otras habilidades propias de la indagación científica como formular preguntas, plantear hipótesis, planificar experimentos y sacar conclusiones.
} 
la recolección y el procesamiento de datos al instante. En el campo educativo se han realizado diferentes investigaciones que buscan establecer su potencial para la enseñanza, por tratarse de una herramienta que puede apoyar el trabajo de laboratorio, en este caso para la Física.

Los SAD están conformados básicamente por un hardware, el cual normalmente está constituido por sensores que captan magnitudes físicas de fenómenos externos y las convierten en señales eléctricas que son acopladas al sistema digital a través de un microprocesador; y por un software que almacena, procesa y muestra dichas señales a través de una pantalla. Con este dispositivo el sujeto puede observar las características y comportamientos de las variables implicadas en el fenómeno. La figura 1 representa las diferentes etapas de un SAD.

Estas etapas dan cuenta de la manera en que el SAD permite medir y representar variables de un determinado fenómeno físico. Cardona y López (2017) explican al respecto que

en la etapa transductora, el transductor o sensor, siendo sensible a esta variable, la transforma en una señal eléctrica que luego ingresa a la etapa de acondicionamiento de señal. En esta etapa la señal es amplificada o filtrada en frecuencias, para luego ser llevada al conversor análogo-digital donde es acoplada al sistema digital. Este sistema, que puede ser un microcontrolador como los presentes en las placas Arduino, puede realizar algún procesamiento de señal o solo enviarla al computador donde se realiza toda la etapa de procesamiento numérico correspondiente a cada experimento particular. (p. 2)

Las ventajas que ofrecen estos dispositivos hacen que el trabajo práctico de laboratorio se convierta en un espacio óptimo de aprendizaje al permitir que los estudiantes enfoquen su actividad en el desarrollo de habilidades metacognitivas mediante procesos de conceptualización (Cardona y López, 2017; Haag et al., 2005).

Se ha reportado en la literatura especializada que con el uso de estas herramientas los estudiantes tienen la oportunidad de realizar actividades a las que no tienen acceso con cierta frecuencia, como hacer el montaje de diferentes experimentos (Grala y de Oliveira, 2005; Garg et al., 2010; Torres, 2010; da Rocha y Marranghello, 2013; Huang, 2015; Kubínová y Šlégr, 2015), conocer el software para el procesamiento de datos (Grala y de Oliveira, 2005; Garg et al., 2010; Chen et al., 2014; Rosa et al., 2016), realizar mediciones en un tiempo más reducido (Sias y Teixeira, 2006; da Silva, 2006; Garg et al., 2010; Chen et al., 2014; Kubínová y Šlégr, 2015), negociar significados con sus compañeros, además de contar con mayor tiempo para interpretar y analizar el desarrollo del trabajo experimental como tal (Sias y Teixeira, 2006; Garg et al., 2010; Andrades et al., 2013; Rosa et al., 2016). Por ello, de acuerdo con Sias y Teixeira: "se trata de un recurso con grandes potencialidades, no solo en la búsqueda de un aprendizaje más significativo por el

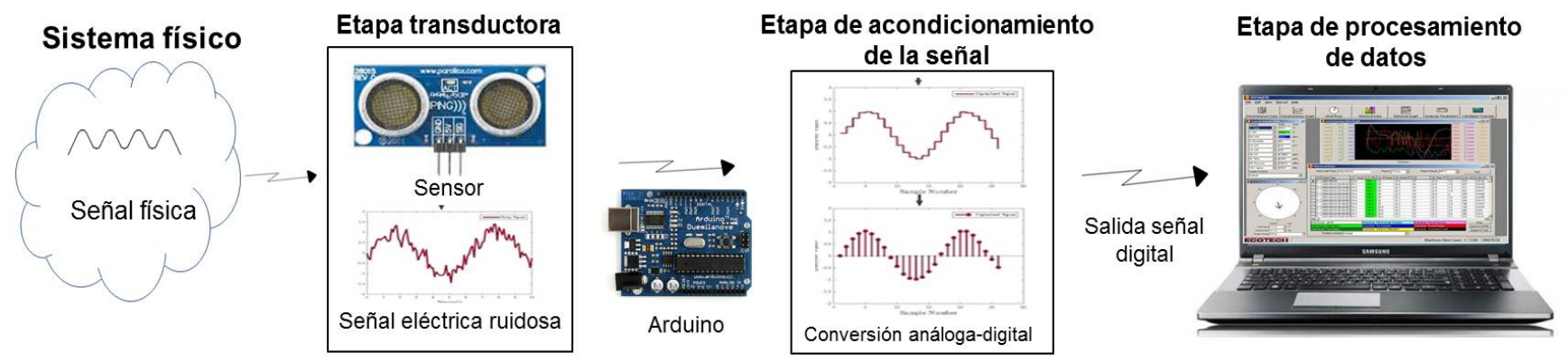

Figura 1. Diferentes etapas de un sistema de adquisición de datos.

Fuentes: (Cardona y López, 2017). 
alumno, sino también como recurso motivador en la discusión de fenómenos físicos" (2006, p. 360).

En particular, los SAD ofrecen la oportunidad de ayudar a mejorar la comprensión de conceptos físicos, siempre y cuando los docentes permitan que los estudiantes tengan más autonomía al interactuar con esta herramienta y adquieran mayor compromiso con su propio aprendizaje (Cavalcante et al., 2008; Moreira y Pontelo, 2009; Novicki et al., 2011). Por este motivo, estos trabajos son una oportunidad para reflexionar sobre la apropiación en general de las TIC por parte de los docentes, ya que son estos los encargados de diseñar estrategias para su implementación en el aula.

Por otra parte, las estrategias de enseñanza utilizadas para apoyar la implementación de estas herramientas tecnológicas tienen un papel importante en los trabajos analizados, pues atienden a la necesidad de generar propuestas que permitan a los docentes en ejercicio y en formación apropiarse del uso de las TIC con criterio pedagógico y didáctico. En este orden de consideraciones es importante tener en cuenta las dificultades que se pueden presentar en dicho proceso, entre las cuales Cavalcante et al. (2008) destacan la falta de preparación para manipular sensores y para el análisis de los datos recogidos por estos mismos, lo que puede generar retrocesos en el proceso de aprendizaje de los estudiantes. La familiarización con estas herramientas es de suma importancia para favorecer la conceptualización y la adquisición de aprendizajes significativos (Amrani y Paradis, 2010).

En general, los hallazgos en relación con la implementación de SAD en los trabajos prácticos de laboratorio permiten identificar las ventajas que estos ofrecen. Sin embargo, son pocos los estudios desarrollados que indagan sobre el papel de estas herramientas en la formación de profesores de Física. Es por esto por lo que el presente artículo tiene como propósito dar cuenta de las ideas que tienen los profesores en formación con respecto al aporte de los SAD a la conceptualización en el trabajo de laboratorio en Física.

\section{Los trabajos prácticos en la enseñanza de las ciencias}

Los trabajos prácticos en la enseñanza de las ciencias han sido objeto de múltiples discusiones (Caamaño, 2004; Barbera y Valdés, 1996; Carrascosa et al., 2006; Ferreiros y Ordóñez, 2002; Cortés y De la Gándara, 2007; Hodson 1994; Malagón et al., 2013; Séré, 2002). Los fundamentos ontológicos, epistemológicos y metodológicos de esta estrategia de aprendizaje han ido cambiando a través de las épocas y se han concentrado en atender al papel que se le ha atribuido en los procesos de enseñanza y aprendizaje.

Para esta investigación los trabajos prácticos están en consonancia con los planteamientos de Caamaño (2007), quien los considera como una actividad de gran importancia en la enseñanza de las ciencias, en la medida que permite:

la familiarización, observación e interpretación de los fenómenos que son objetos de estudio en las clases de ciencias, el contraste de hipótesis en los procesos de modelización de la ciencia escolar, el aprendizaje del manejo de instrumentos y técnicas de laboratorio y de campo, la aplicación de estrategias de investigación para la resolución de problemas teóricos y prácticos y, en definitiva, la comprensión procedimental de la ciencia. (p. 95)

Por otra parte, es importante anotar que los trabajos prácticos pueden variar atendiendo a los objetivos que se pretendan alcanzar mediante su implementación. Caamaño (2004) concibe que esta estrategia de aprendizaje puede llevarse a cabo bajo las siguientes modalidades: experiencias, experimentos ilustrativos, ejercicios prácticos e investigaciones.

La modalidad de trabajos prácticos como investigación es valorada positivamente por varios autores (Caamaño, 2004; Carrascosa et al. 2006; Cortés y De la Gándara, 2007), pues permite al estudiante trabajar en torno a la solución de problemas específicos y no mediante una guía predeterminada 
como suele plantearse normalmente. En el presente estudio se asume esta última modalidad de trabajos prácticos por estar en consonancia con algunos de los planteamientos de la teoría de los campos conceptuales, la cual considera, entre otras cosas, que los procesos de aprendizaje se llevan a cabo cuando los sujetos ponen en juego sus esquemas para hacer frente a las situaciones que se les presenta. Para este caso, dichas situaciones están enmarcadas en el contexto del trabajo práctico de laboratorio de Física.

\section{La teoría de los campos conceptuales en la enseñanza de las ciencias}

La teoría de los campos conceptuales está sustentada en planteamientos teóricos de Piaget, de quien Vergnaud retoma el concepto de esquema; $y$ desde Vygotsky, en lo relativo al sentido de la mediación a través del lenguaje. Su interés está centrado principalmente en analizar la forma en que los sujetos construyen conceptos.

En el ámbito de la educación en ciencias, usualmente se concibe que la apropiación de un concepto se refiere a la capacidad que tiene el sujeto para definirlo en términos científicos, o en el mejor de los casos, de relacionarlo con sus concepciones previas. Desde esta teoría cognitiva, el proceso de conceptualización trasciende notablemente esta noción de aprendizaje. Los conceptos cobran sentido para el sujeto solo cuando son puestos en situaciones que forman parte de un determinado campo conceptual, que es para Vergnaud (1983) "un conjunto informal y heterogéneo de problemas, situaciones, conceptos, relaciones, estructuras, contenidos y operaciones del pensamiento, conectados unos a otros y, probablemente, entrelazados durante el proceso de adquisición" (como se cita en Moreira, 2002, p. 2).

Dado que esta es la forma en que se organiza el conocimiento, el aprendizaje de los sujetos se valora en torno a la apropiación de los diferentes elementos que subyacen a un campo conceptual y a las relaciones entre ellos. En este sentido, la conceptualización se entiende como un proceso que implica la interacción permanente entre las situaciones, que se definen como una combinación de tareas que involucran procesos cognitivos complejos; y los esquemas, que se entienden como "la organización invariante de la conducta para una clase de situaciones dada" (Vergnaud, 1990, p. 2). Entre los elementos cognitivos de los esquemas se encuentran las metas, reglas de acción, inferencias e invariantes operatorios. Estos últimos tienen la función de identificar las propiedades, relaciones y transformaciones de los objetos, seleccionar la información pertinente para establecer las reglas de acción que permitirán abordar una situación (junto con los conceptos y las representaciones) para que de esta manera el grado de dominio para enfrentar dichas situaciones es el reflejo de la propia conceptualización.

Dado que los invariantes operatorios son considerados conocimientos implícitos que un estudiante tiene y se le dificulta explicitar, es a través de las representaciones (lenguaje natural, gráficos y diagramas, sentencias formales, entre otros) que podría lograrse que un sujeto exteriorice los conceptos que ha construido y los procedimientos que utiliza para abordar las situaciones propuestas. Es decir, a través de las representaciones se manifiestan los conocimientos que permiten seleccionar la información necesaria para enfrentar una situación. Desde esta perspectiva, y en el ámbito de este trabajo, se considera que los docentes actúan como mediadores que utilizan expresiones para explicar, preguntar, proponer metas, reglas, entre otros; favoreciendo a través del lenguaje la construcción de representaciones en los estudiantes. Lo anterior permite que los sujetos enriquezcan sus esquemas, adquieran un mayor dominio de las situaciones a las que se enfrentan y, por ende, se vea favorecido su proceso de conceptualización.

\section{Metodología}

El trabajo tuvo un enfoque constructivista enmarcado en el paradigma cualitativo. Desde esta 
perspectiva se seleccionó el estudio de caso instrumental como método de investigación (Stake, 2010). Para este trabajo el caso estuvo constituido por un grupo de profesores en formación del área de Ciencias Naturales, del cual fueron estudiadas sus concepciones en relación con el aporte de los SAD a los procesos de conceptualización al realizar un trabajo práctico de laboratorio en Física.

El presente estudio se llevó a cabo en la Facultad de Educación de la Universidad de Antioquia, Colombia, teniendo como participantes un grupo de ocho profesores en formación del área de Ciencias (identificados como E1 a E8) que se encontraban cursando la asignatura de Física II. Este curso forma parte del componente disciplinar de la Licenciatura en Ciencias Naturales y Educación Ambiental. La propuesta de enseñanza tuvo como propósito identificar las principales características del trabajo de laboratorio orientado con SAD durante la formación de profesores de Ciencias. No obstante, para efectos del presente artículo, se hará énfasis en la valoración de los participantes a la contribución de esta herramienta en el trabajo práctico de laboratorio en Física haciendo uso de SAD.

La propuesta se diseñó a partir del modelo de aprendizaje en los trabajos de laboratorio de Física MATLaF (Andrés et al., 2006), el cual fue elaborado para identificar el desarrollo cognitivo de los participantes al enfrentarse a situaciones experimentales novedosas. A partir de este modelo se llevaron a cabo tres momentos con propósitos definidos para la recolección de la información que se sintetizan en la figura 2.

Como se observa en la figura 2, dichos momentos siguieron un plan de acción que se compuso de cuatro fases, las cuales se llevaron a cabo durante ocho sesiones (cinco sesiones de cuatro horas cada una y tres sesiones de dos horas). Para un total de 26 horas, durante de aproximadamente un mes.

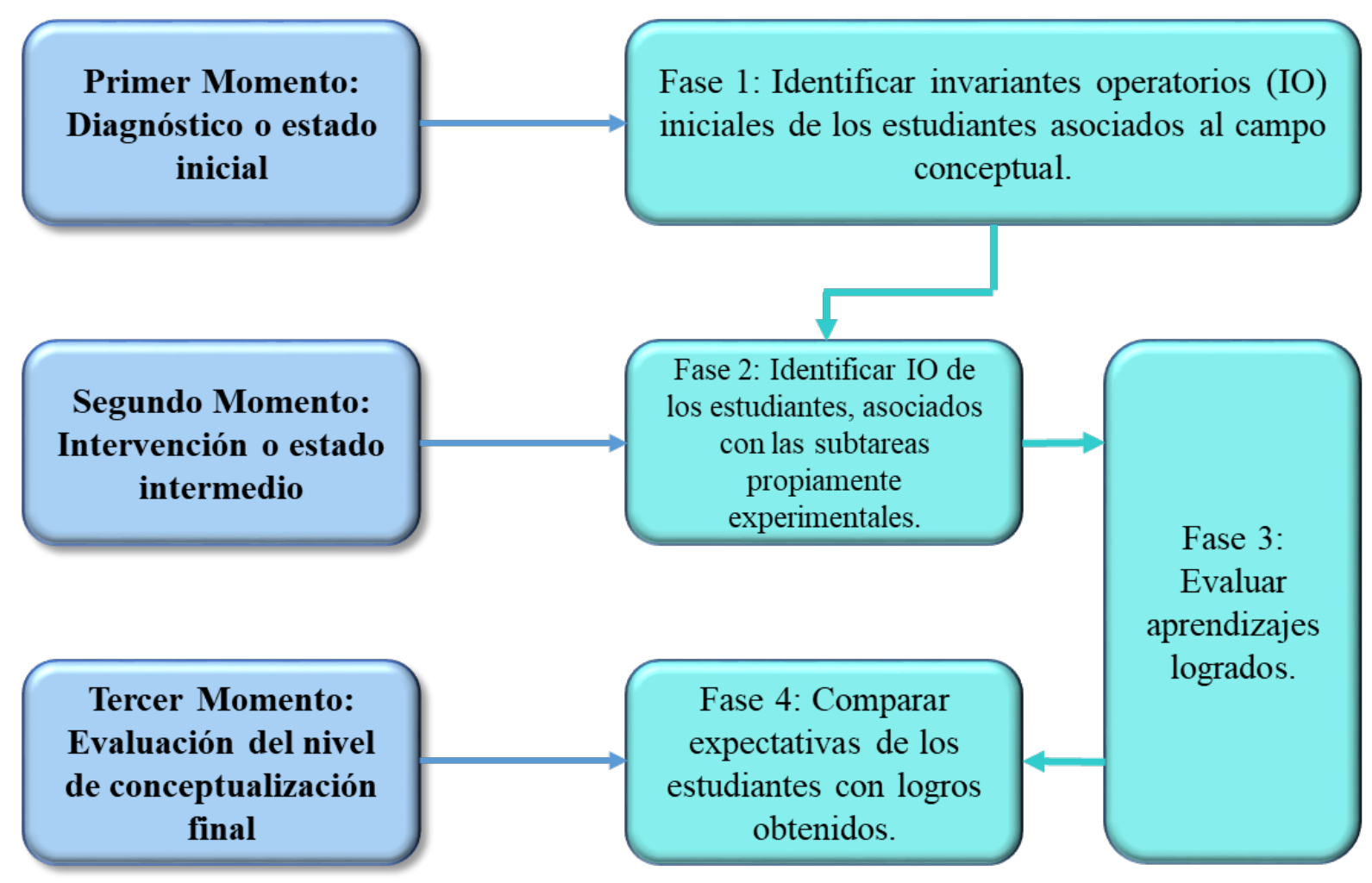

Figura 2. Momentos y fases de la propuesta de enseñanza.

Fuente: elaboración propia de los autores. 
El primer momento (una sesión de dos horas) comenzó con la observación de experimentos en los que se involucró la generación de un campo magnético. En este se discutieron concepciones previas de los participantes que dieron una explicación sobre tres fenómenos que observaron: la interacción entre materiales magnéticos y no magnéticos; el comportamiento de un imán polarizado acomodado en la superficie del agua; y un motor eléctrico diseñado a partir de una pila, imanes y alambre de cobre. Posteriormente, se realizó una discusión que estuvo mediada por preguntas abiertas. Al final de este primer momento se realizó una presentación a los participantes sobre los SAD, explicando en qué consistían y mostrando diferentes tipos de sensores y su utilidad (Cardona, 2018a)

El segundo momento se realizó durante seis sesiones (cinco sesiones de cuatro horas y una de dos horas) en las que se desarrollaron tres trabajos de laboratorio, exposiciones de los participantes e intervenciones del docente siguiendo la programación regular del curso. Se pusieron en marcha diferentes estrategias como el aprendizaje colaborativo, una adaptación del diagrama $\mathrm{V}$ de Gowin y la bitácora personal (Cardona, 2018b, pp. 177-193). El aprendizaje colaborativo involucró a los participantes en la construcción de conocimiento a través de la interacción con sus pares, lo que también posibilitó desarrollar las capacidades necesarias para la interacción social, que, según Vygotsky, son un medio fundamental para la adquisición de significados acordes con el contexto. Las diferentes actividades que conforman el segundo momento de la propuesta didáctica son descritas a continuación.

\section{Actividad 1. Caracterización de un campo magnético generado por un imán permanente (una sesión de tres horas)}

Para esta actividad se usaron como materiales: imanes, brújulas, papel polar, lápiz, sensor de efecto Hall A1302, microprocesador Arduino (Chipkit uno32) y la bitácora con algunas preguntas para ser desarrolladas de manera individual. Cada grupo contaba con un computador de escritorio en el que previamente se instaló un programa diseñado en Processing para la visualización de los datos. En la figura 3 se observa parte del desarrollo de esta actividad.

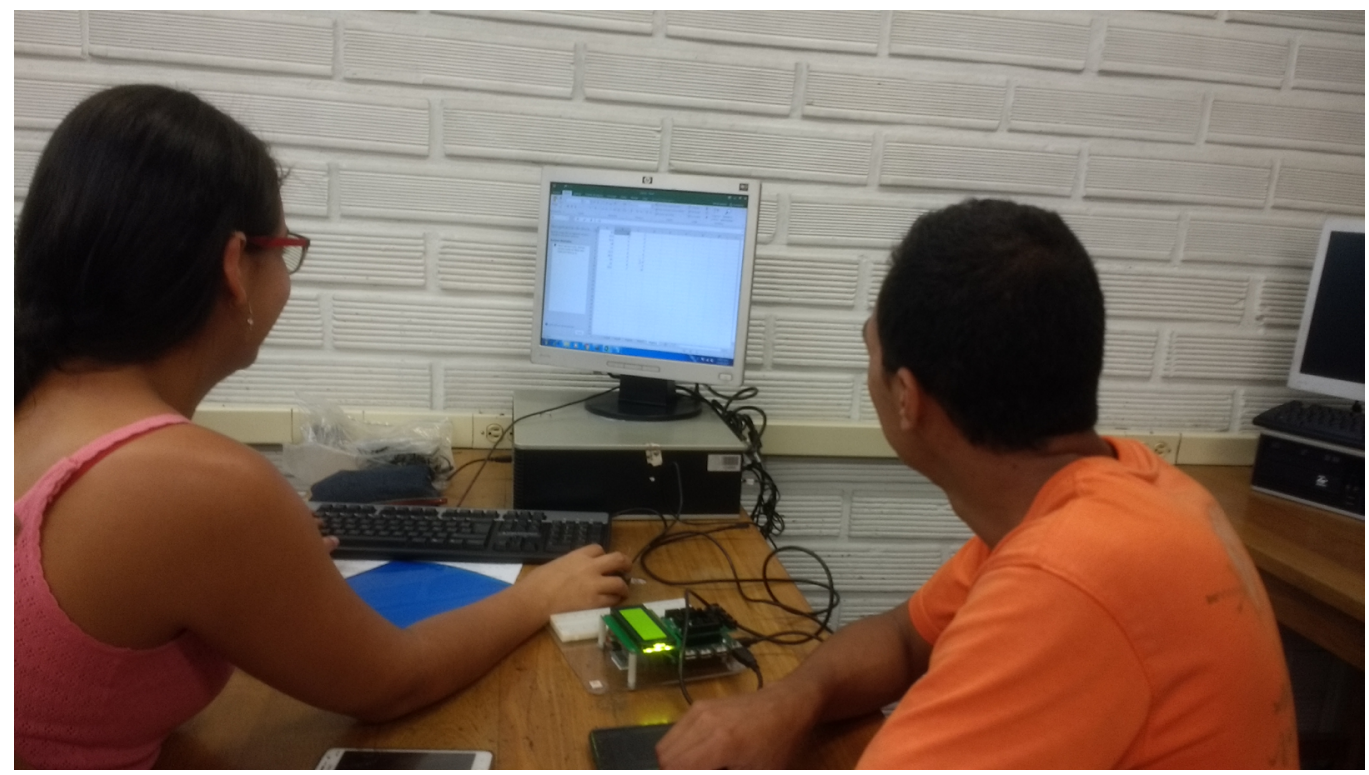

Figura 3. Caracterización del campo magnético con la mediación de SAD.

Fuente: elaboración propia de los autores. 
El objetivo de este ejercicio práctico consistía en identificar la variación del campo magnético de un imán permanente con la distancia y su relación con la forma del imán. Este se orientó a partir de las preguntas: ¿cómo varía el campo magnético con la distancia en los imanes permanentes? y ¿Cómo afecta esa variación dependiendo de la forma del imán?

\section{Actividad 2. Medir el campo magnético en el centro de una bobina (una sesión de dos horas)}

Para esta actividad los participantes se organizaron en grupos de trabajo y utilizaron como materiales: bobina, fuente de corriente, cables banana-caimán, sensor de efecto Hall A1302, microprocesador Arduino (Chipkit uno32), barra de acero, voltímetro y computador. El trabajo de laboratorio estuvo apoyado por los investigadores, el docente del curso y un docente de Física quien fue acompañante. El objetivo era identificar la respuesta de un material ferromagnético frente a la aplicación de un campo magnético externo y se orientó a partir de las preguntas: ¿cómo se relaciona el campo magnético dentro de una bobina con la corriente que circula por esta? y ¿cómo cambia el campo magnético al interior de la bobina si se introduce en su núcleo un material ferromagnético?

\section{Actividad 3. Medición de la velocidad angular de un motor eléctrico conformado por una batería, un imán y un alambre conductor (una sesión de dos horas)}

El último trabajo de laboratorio se diseñó atendiendo a la programación regular del curso con el fin de complementar la temática que adelantaba el docente en sus clases. Por este motivo se optó por elaborar una situación con el propósito de que los participantes identificaran la relación entre la velocidad angular de un motor eléctrico simple y el campo magnético generado por el imán que lo conformaba. Para esta actividad los participantes diseñaron un motor eléctrico a partir de una pila, un imán y alambre de cobre; para la recolección de datos utilizaron una fotocompuerta marca Pasco, un microprocesador Arduino (Chipkit uno32), el software Hyper serial port y un calibrador (pie de rey) para medir el diámetro de la espiral del motor. En la figura 4 se observa el montaje realizado por los participantes.

Para el abordaje de la situación planteada, los participantes estuvieron orientados a partir de las preguntas: ¿cuál es el papel del campo magnético en el funcionamiento del motor?, ¿qué implicación tiene la variación del campo magnético en el movimiento del motor? y ¿por qué?

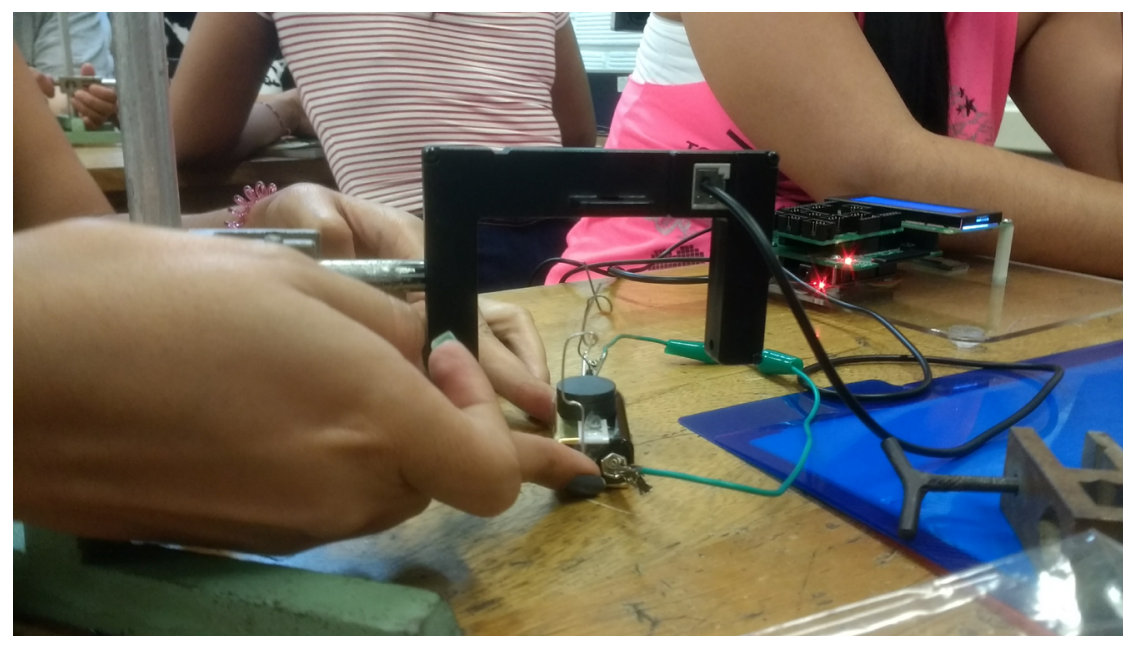

Figura 4. Montaje para la medición de la velocidad angular de un motor eléctrico.

Fuente: elaboración propia de los autores. 
Para cada una de las situaciones ya presentadas los participantes describieron en su bitácora el procedimiento para abordarla, así como sus ideas, reflexiones, decisiones, entre otros aspectos que permitieron reflejar el proceso de conceptualización. En dichas actividades utilizaron la adaptación del diagrama $V$ de Gowin, entendiendo que durante el trabajo práctico de laboratorio predomina "el aprendizaje del dominio metodológico en interrelación con algún marco teórico de referencia asociado a la situación planteada" (Andrés et al., 2006, p. 134). Luego de cada actividad se socializaron los resultados obtenidos por los participantes, comparándolos con los resultados esperados y aclarando inquietudes a nivel conceptual.

Sobre el tercer momento correspondiente a la evaluación de los aprendizajes logrados se debe resaltar que tuvo como propósito identificar el nivel de conceptualización de los participantes a través de los productos, las exposiciones, las discusiones y los registros en la bitácora personal. En la última sesión (dos horas) los participantes se enfrentaron a una pregunta sobre diferentes fenómenos que involucraban el electromagnetismo como campo conceptual y discutieron en grupo su comprensión desde el punto de vista físico de cada fenómeno y su aplicabilidad en diferentes contextos. Cada pregunta contaba con un texto breve sobre la aplicación o el fenómeno involucrado y dos interrogantes para generar la discusión entre los participantes de cada grupo: desde el punto de vista físico, ¿cómo explican el fenómeno? y ¿qué papel cumple el fenómeno en las aplicaciones mencionadas? Luego cada equipo socializó de manera breve la explicación de cada fenómeno enfatizando en su relación con el concepto de campo magnético. Por último, se realizó la entrevista individual semiestructurada a los participantes con el propósito de conocer sus posturas sobre el nivel de conceptualización alcanzado.

\section{Resultados}

A partir del análisis de diferentes instrumentos como la bitácora de los participantes, el diario de campo de los investigadores y la entrevista individual final semiestructurada, fue posible identificar principalmente dos tipos de concepciones de los profesores en formación sobre el aporte de los SAD a la conceptualización en el trabajo práctico de laboratorio. A saber: los SAD como instrumento de medición y los SAD para favorecer la representación de fenómenos físicos.

\section{SAD como instrumento de medición}

En esta categoría se identificaron expresiones de los participantes que hacen referencia al uso de SAD como instrumento de medición. Se resaltan en particular las respuestas obtenidas al indagar por su experiencia con los SAD durante la propuesta, así como por las diferencias que se encontraron entre los trabajos prácticos de laboratorio llevados a cabo y otras experiencias anteriores:

- Pues yo digo que, desde la instrucción, del modelo, por ejemplo, un laboratorio de física como lo que hicimos con los sensores. Pues, uno sí empezaba a jugar por ejemplo con el voltímetro, pero uno entendía el sentido de las cosas. Uno sabía que, si por ejemplo uno tenía la barra de acero dentro, eso te generaba unos valores diferentes; sabías que el campo estaba aumentando, disminuyendo. (E2)

- Hubiese sido interesante interactuar también con todo lo que el sensor hace; porque era simplemente conectarlo al computador y sí muy bacano los datos que arrojaba, todo eso se ve muy interesante, porque era la primera vez que muchos veían algo así. (E5)

- No había tenido un contacto con ese tipo de tecnología y ese tipo de manera experimental y de recolección de datos para hacer análisis y comprobarlo con las teorías [...] los experimentos y todos los procesos que hicimos en el laboratorio, era una parte que era de la toma de datos y todo esto que era lo que correspondía al sensor y los materiales que a nosotros nos daban. (E8)

- Cuando estábamos con el motor eléctrico, que debíamos medir lo de las oscilaciones que 
tenía el motor, cuando arrojaba las gráficas ya uno como que determinaba ah bueno, entonces si lo comparo con la teoría, me va a decir si lo hicimos bien y si el sensor si funciona, entonces yo creo que eso fue lo más significativo que hubo ahí, con respecto a eso. (E6)

- Veíamos cómo pasábamos el sensor y eso ya daba muchos datos. (E4)

- Pero es que yo lo veo como, pues los datos llegan ahí, cuando uno conecta el cable al computador, pues eso ya todo se transfiere al computador, simplemente para hacer la gráfica, es como un intermediario, creería yo, pues yo lo veo así. (E5)

En las expresiones subrayadas es clara la noción de los participantes sobre la relación entre teoría y práctica, pues aún expresan sin criterios claros que los datos se utilizan para comprobar la teoría; en particular en la forma como es manifestada por los participantes E6 y E8. En las respuestas de los demás se identificó un evidente interés por explorar dicha herramienta por su novedad para apoyar el trabajo de laboratorio y por la comprensión que tuvieron de los fenómenos a partir de su uso. También se resalta su opinión sobre el favorecimiento de la recolección y procesamiento de los datos, lo que les permitió establecer relaciones entre las magnitudes medidas.
Por ejemplo, en la figura 5 se presenta la gráfica obtenida en la Actividad 3 del segundo momento de la propuesta, a partir de la cual los participantes registraron el tiempo que tardaba la espira del motor construido en dar una vuelta y pasar nuevamente a través de la fotocompuerta.

En la bitácora de los participantes se identificaron algunos fragmentos que hacen alusión a los potenciales usos de los SAD durante esta actividad, destacando los siguientes:

- La fotocompuerta nos permitió determinar el tiempo en el que se cruzaba el aro o círculo, es decir, la "eficiencia" del motor. (E5)

- La fotocompuerta mide el tiempo que tarda en dar una vuelta la espira del motor en un segundo, tal información es recogida por el Arduino y llevada al computador. (E7)

- El uso de dispositivos electrónicos como el Arduino, pueden generar la adquisición de un conocimiento significativo en múltiples áreas, a partir de la adquisición de datos en tiempo real sobre un fenómeno que se experimenta directamente. (E7)

Estas características sobresalen por encima del uso de otras herramientas pues favorecen mediciones que tendrían un grado mayor de dificultad con instrumentos convencionales. Lo que, de acuerdo

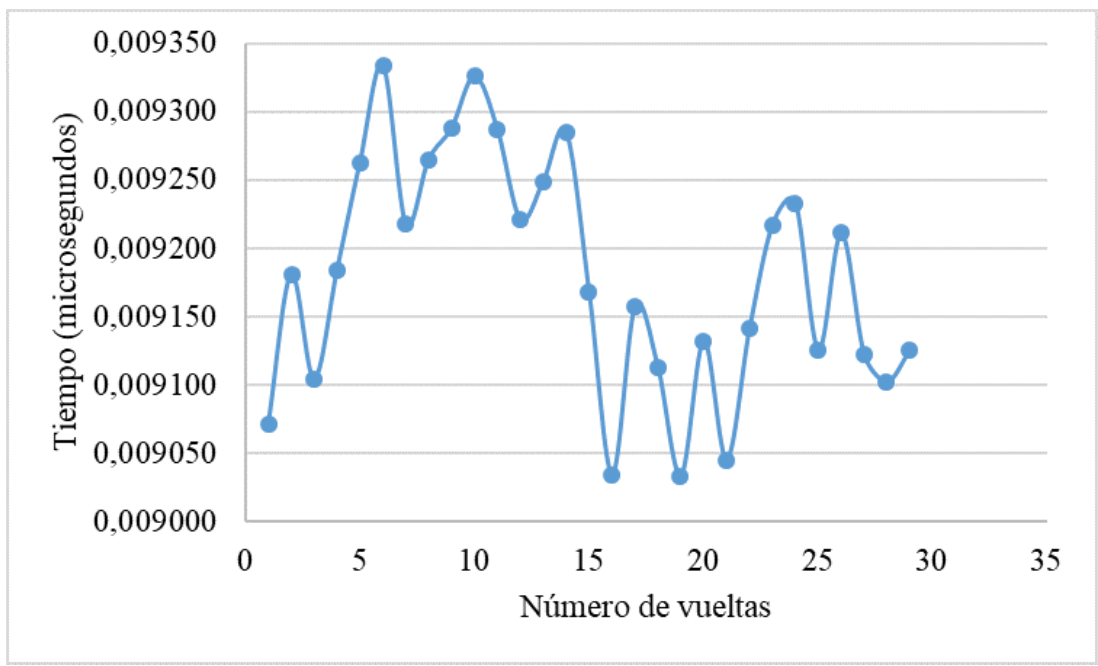

Figura 5. Gráfica del tiempo de rotación del motor por cada vuelta.

Fuente: elaboración propia de los autores. 
con da Silva (2006): "posibilita que el alumno adquiera algunas nociones sobre técnicas de medida contemporáneas y tecnologías empleadas en el día a día" (p. 19). De igual manera, desde la teoría de los campos conceptuales, los SAD como instrumentos de medición hacen parte del dominio metodológico de los trabajos prácticos, lo que según Andrés et al. (2006) está "en interrelación indisoluble con algún marco teórico de referencia asociado a la situación planteada" (p. 134). Es decir, la caracterización propuesta por los participantes del papel de dicha herramienta es coherente con el favorecimiento de los procesos de conceptualización en los participantes.

Es importante anotar la posibilidad de involucrar esta herramienta en este espacio de aprendizaje. Si el trabajo de laboratorio se realiza en el marco de situaciones que requieren el enfrentarse a diversos tipos de tareas, los SAD como instrumentos de medición pueden ayudar notablemente a los participantes en el abordaje de dichas situaciones que necesitan de una compleja actividad cognitiva.

T2SAD para favorecer la representación

Para presentar la discusión de los resultados en esta categoría, se resaltan los siguientes fragmentos de la bitácora de los participantes, desde la pregunta sobre ¿qué reflexiones surgen a partir de esta experiencia?, realizada en el primer trabajo de laboratorio:

- En la experiencia de aprendizaje con los sensores y los imanes, entendemos que estos poseen campos. (E2)

- La forma que tiene cada imán determina la curvatura de la gráfica resultante de los datos obtenidos por el sensor de campo magnético conectado al Arduino, además tal gráfica permite inferir que entre más cerca se está del imán, más fuerte es el campo magnético. (E7)

Los anteriores enunciados permiten observar que durante esta actividad el uso de los sensores influyó en el primer acercamiento que tuvieron los participantes a la caracterización del campo magnético generado por un imán. Actividad que también se complementó con el desarrollo del diagrama $V$, del cual se resaltan las siguientes afirmaciones:

- Según las gráficas realizadas por el Arduino de campo vs. distancia concuerdan con la teoría, las gráficas muestran puntos más alejados en cuanto se aleja más el sensor del imán, también se evidencia un equilibro después de colocar el sensor en otros puntos diferentes del más cercano a él. (E1)

- El estudio del campo magnético nos proporciona datos que permiten un conocimiento más amplio acerca de la interacción con el imán. (E6)

- Se observa un cambio de las líneas de campo detectadas por el sensor al variar la distancia. (E8)

- Los resultados obtenidos a partir de los datos adquiridos por el Arduino verifican una velocidad constante en la velocidad del motor, la cual se verá alterada por la modificación del campo magnético aumentando o disminuyendo la rotación de la espira. (E2, E3, E7)

En estas afirmaciones los participantes se refieren a características de los fenómenos observados, como la variación del campo con la distancia, las líneas de campo y la variación de la velocidad angular del motor, las cuales fueron identificadas gracias al uso de los sensores. Se reconoció así que con esta herramienta fue posible distinguir el comportamiento del campo magnético alrededor de un imán, así como su influencia en el funcionamiento del motor. Durante la entrevista semiestructurada se indagó a los participantes sobre ¿cómo fue su experiencia con los SAD durante la intervención?, ¿qué diferencias encuentra entre las actividades realizadas y sus experiencias anteriores? y ¿cómo valora su proceso de aprendizaje del concepto de campo magnético a través de esta propuesta? A partir de las respuestas obtenidas, se identificaron algunos puntos de encuentro que se describen en la tabla 1. 
Tabla 1. Características de los SAD para favorecer la representación, identificadas en la entrevista semiestructurada.

\section{Respuestas de los participantes}

Aparte de que uno conoce un montón de conceptos que están en la teoría [...] los puede realmente, más o menos como sentir [...] por ejemplo esto del campo magnético, utilizado sensores para saber que ahí hay algo. (E7)

Con respecto a lo que estábamos viendo de los sensores, estábamos ahí en el momento, teníamos los sensores, los podíamos manipular, podíamos usarlos y eso para mí fue significativo. (E6)

Si no hubieran estado los sensores hubiera sido mucho más complicado de entender ese concepto, porque no había algo que nos graficara todo [...] entonces por ejemplo [...] ¿cómo hubiéramos explicado eso? [...] porque entonces el estudiante no tenía una representación de las líneas de campo, de, por ejemplo, qué generaba si se aumentaba la corriente [...] no había una forma de representar lo que daba, entonces para eso sirvió mucho los sensores. (E1)

Pues yo creo que uno sí se defiende más, pues si hay un cambio. No sé en lo matemático, pero en lo conceptual sí; como esa mezcla entre lo teórico y lo práctico y cómo representarlo [...] porque eso ayuda a ver [...] a tener una referencia de por qué pasa y cómo lo puede explicar. (E3)

Los numeritos que nos daba en el computador, pues era más creíble y nos hacía comprender y darnos cuenta que había algo que estaba sucediendo ahí aunque no lo estuviéramos viendo. (E5)

Eso empezaba una línea recta y después como un pico [...] Entonces uno se ponía a revisar y veía jah no! fue que acá cambió el campo, o le metimos más voltaje, entonces por eso daba unos picos tan extraños. (E2)

Cuando empezamos a usar los sensores, que veíamos ya una relación y que podíamos sacar los datos para hacer las gráficas, uno decía esto sí sirve, esto sí representa una diferencia y simplificaría mucho el aprendizaje y lo haría mucho más entendible. (E2)

También por las gráficas podíamos entender cuándo disminuía, cuándo era más fuerte, cuándo era más débil el campo, entonces claro eso nos ayudó. (E5)

[...] esos resultados que por ejemplo en el laboratorio nos daban, pues ya nos dábamos cuenta después que eso se podía graficar y que esos resultados iban a tener sentido porque ya la gráfica con lo que teníamos de teoría decíamos jah sí! está decreciendo... es que si tenía que decrecer. Me parece muy importante que las gráficas se hagan porque es una forma de darle ese significado a esos números. (E5)

Uno ya sabiendo analizar una gráfica, puede tomar los mismos datos, obtenerlos con el Arduino y analizar la gráfica; entonces si uno no monta un experimento con un sistema de adquisición de datos es más difícil uno analizarlo realmente, uno, pues solo se basa en la teoría que lo sustente. Creo que es mucho mejor y que cambia drásticamente un laboratorio el uso de esos aparatos. (E7)

Estar frente a esas cosas que normalmente no se pueden usar o tocar porque son o caros o porque no los sabemos usar [...]. Aparte de que la última clase que hicimos [...] eran temas que ya conocíamos, porque hicimos la práctica, hicimos las exposiciones... entonces llegar a compactarlo con lo que hicimos en la última clase que fue exponer esos temas, ya uno como que jah bueno! yo ya tengo conocimiento de lo que es un motor eléctrico o cómo funcionan los campos magnéticos. (E6)

Nos abre un poquito más las metodologías porque obviamente son metodologías nuevas [...] que se pueden implementar y que pueden servir porque son un poco más abiertas. Le exigen al estudiante [...] en el campo en el que se están formando, antes del laboratorio, durante los procedimientos y después de los procedimientos, y después van a enlazar todos los contenidos. (E8)

\section{Característica de los SAD}

Favorece la representación de un fenómeno involucrando diferentes sentidos.

Apoya la interpretación de gráficas obtenidas a partir de los datos recolectados y la relación entre variables

Permite comparar diferentes situaciones que involucran el fenómeno

Fuente: elaboración propia de los autores. 


\section{Conclusiones}

En este trabajo se encuentran importantes resultados que pueden ser útiles para profesores de Física en formación y en ejercicio, en relación con el papel de los SAD para el trabajo práctico de laboratorio. Los resultados dan cuenta de que la implementación de SAD, orientada desde la teoría de los campos conceptuales, ayuda a transformar la imagen técnico-instrumental de la enseñanza de la Física puesto que los estudiantes manifiestan que la recolección de datos con esta herramienta no tuvo un carácter meramente instrumental, sino que fue una tarea que les ayudó a reflexionar sobre la forma como se construyen los conceptos a partir de la experimentación. Al analizar las concepciones de los futuros profesores en relación con el uso de SAD, se identificaron principalmente dos características: 1) su uso como instrumento de medición apoyó la interpretación de gráficas obtenidas a partir de los datos recolectados y la relación entre variables y 2) al favorecer la representación se logró tener una visualización más general e inmediata de los sistemas físicos reales.

A partir de las expresiones de los participantes, con respecto a los SAD como instrumento de medición, se pudo valorar el apoyo de estos dispositivos tecnológicos a los trabajos prácticos desde tres puntos de vista: la sofisticación tecnológica, la perspectiva epistemológica y las contribuciones al aprendizaje. Al ser dispositivos tecnológicos avanzados, las posibilidades que ofrecen los SAD desde el punto de vista técnico fueron valoradas de manera positiva por los participantes. Se resaltaron características como la velocidad de procesamiento de datos, la precisión con la que se obtenían estos y la relación en tiempo real que permitieron hacer de las variables implicadas en el fenómeno físico. Asimismo, los participantes manifestaron que estos dispositivos permitían evidenciar de una manera más clara la relación teoría-práctica. Lo anterior parece estar en correspondencia con el hecho de que los elementos que subyacen al funcionamiento del SAD solo tienen validez a la luz de una teoría (e. g., electromagnetismo), y sentido a partir de una situación que requiere de su uso (e. g., medir el campo magnético).

Por último, con el uso de este recurso se favoreció un eje fundamental de la teoría que enmarca esta investigación y es la representación, entendida como la manera que tiene un sujeto para comunicar lo que está presente en sus esquemas. Ya que, como se describió en el análisis de resultados, el uso de SAD favoreció la representación de los fenómenos involucrando diferentes sentidos; apoyó la interpretación de gráficas obtenidas a partir de los datos recolectados y la relación entre variables, lo que permitió comparar diferentes situaciones que involucran un fenómeno.

\section{Referencias}

Amrani, D., Paradis, P. (2010). Use of Computer-Based Data Acquisition to Teach Physics Laboratories: Case study-Simple Harmonic Motion. Latinoamerican Journal of Physics Education, 4(3), 511-514.

Andrades, J., Schiappacassa, A., Santos, P. (2013). Development of a microcontrolled periodmeter for experimental physics applications. Revista Brasileira de Ensino de Física, 35(2), 1-11. https://doi.org/10.1590/ $\underline{\text { S1806-11172013000200023 }}$

Andrés, M., Pesa, M., Moreira, M. (2006). El trabajo de laboratorio en cursos de física desde la teoría de los campos conceptuales. Ciência \& Educação. Bauru, 12(2), 129-142. https://doi. org/10.1590/S1516-73132006000200002

Ayala, M., Malagón, J., Sandoval, S. (2011). Magnitudes, medición y fenomenologías. Revista de Enseñanza de la Física, 24(1), 43-54.

Barbera, O., Valdés, P. (1996). El trabajo práctico en la enseñanza de las ciencias: una revisión. Enseñanza de las ciencias: revista de investigación y experiencias didácticas, 14(3), 365-379.

Caamaño, A. (2004). Experiencias, experimentos ilustrativos, ejercicios prácticos e 
investigaciones: ¿una clasificación útil de los trabajos prácticos? Alambique, 39(8), 19.

Caamaño, A. (2007). El trabajo práctico en ciencias. En M. P. Jiménez, A. Caamaño, A. Oñorbe y A. de Pro Bueno, Enseñar Ciencia (pp.95118). Graó.

Cardona, M. y López S. (2017). Una revisión de literatura sobre el uso de sistemas de adquisición de datos para la enseñanza de la física en la educación básica, media y en la formación de profesores. Revista Brasileira de Ensino de Física, 39(4), 1-11. https://doi. org/10.1590/1806-9126-rbef-2016-0308

Cardona, M. (2018a). Sistemas de Adquisición de Datos: uso de TIC como apoyo a la actividad experimental en la formación de profesores de Ciencias Naturales. https://view. genial.ly/5b81eac2c1e5736ddb2df44a/ interactive-content-sad

Cardona, M. (2018b). La actividad experimental apoyada en el uso de sistemas de adquisición de datos: una propuesta teórico metodológica para favorecer la conceptualización en física (Trabajo de investigación de maestría). Universidad de Antioquia, Colombia.

Carrascosa, J., Gil, D., Vilches, A., Valdés, P. (2006). Papel de la actividad experimental en la educación científica. Caderno Brasileiro de Ensino de Física, 23(2), 157-165.

Cavalcante, M., Bonizzia, A., Gomes, L. (2008). Aquisiçao de dados em laboratórios de fisica: um método simples, fácil e de baixo custo para experimentos em mecânica. Revista Brasileira de Ensino de FIsica, 30(2), 1-6. https:// doi.org/10.1590/S1806-11172008000200011

Chen, S., Chang, W., Lai, C., Tsai, C. (2014). A Comparison of Students' Approaches to Inquiry, Conceptual Learning, and Attitudes in Simulation-Based and Microcomputer-Based Laboratories. Science Education, 98(5), 905-935.

Cortés, Á. L., De la Gándara Gómez, M. (2007). La construcción de problemas en el laboratorio durante la formación del profesorado: una experiencia didáctica. Enseñanza de las ciencias: revista de investigación y experiencias didácticas, 25(3), 435-450.

da Rocha, F., Marranghello, G. (2013). Propriedades de um acelerômetro eletrônico e possibilidades de uso no ensino de mecânica. Latin-American Journal of Physics Education, 7(1), 37-46.

da Silva, L. (2006). Uma experiência didática com aquisição automática de dados no laboratório de física do ensino médio. Experiências em Ensino de Ciências, 1(3), 18-32.

Ferreirós, J., Ordóñez, J. (2002). Hacia una filosofía de la experimentación (Towards a Philosophy of Experiment). Crítica: Revista Hispanoamericana de Filosofía, 47-86. https:// doi.org/10.22201/iifs.18704905e.2002.979

Garg, A., Sharma, R., Dhingra, V. (2010). Computer assisted magnetism studies. Latinoamerican Journal of Physics Education, 4(3), 523-528.

Grala, R., de Oliveira, E. (2005). Física na Escola, 6(2), 26-28. https://doi.org/10.5216/rpp.v2i0.152

Haag, R., Araujo, I., Veit, E. (2005). Por que e como introduzir a aquisição automática de dados no laboratório didático de física? Física na Escola, 6(1), 69-74.

Hodson, D. (1994). Hacia un enfoque más crítico del trabajo de laboratorio. Enseñanza de las Ciencias, 12(3), 299-313.

Huang, B. (2015). Open-source Hardware -Microcontrollers and Physics Education- Integrating DUY Sensors and Data Acquisition with Arduino. 122nd ASEE Annual Conference \& Exposition (Paper ID \#14073). Seattle: American Society for Engineering Education.

Kubínová, Š., Šlégr, J. (2015). Physics demonstrations with Arduino board. Physics Education, 50(4), 472-474. https://doi. org/10.1088/0031-9120/50/4/472

Malagón-Sánchez, J. F., Sandoval-Osorio, S., Ayala-Manrique, M. M. (2013). La actividad experimental: construcción de fenomenologías y procesos de formalización. Praxis Filosófica, 36. https://doi.org/10.25100/pfilosofica.v0i36.3467 
Moreira, A., Pontelo, I. (2009). Níveis de engajamento em uma atividade prática de Física com aquisição automática de dados. Revista Brasileira de Pesquisa em Educação em Ciências, 9(2), 148-167.

Moreira, M. (2002). La teoría de los campos conceptuales de Vergnaud, la enseñanza de las ciencias y la investigación en el área. Investigaciones en Enseñanza de las Ciencias, 7(1), 1-28.

Novicki, A., da Silveira, E., Poglia, R. (2011). Determinação da velocidade de uma fonte sonora através da aquisição automatica de dados, baseado no efeito doppler-fizeau. Física na Escola, 12(1), 4-7.

Rosa, P., Silva, F., Benyosef, L., Papa, A. (2016). Desenvolvimento de instrumentos virtuais para obtenção e caracterização de propriedades físicas. Caderno Brasileiro de Ensino de Física, 38(1), 1-8. https://doi.org/10.1590/ $\underline{\text { S1806-11173812015 }}$

Séré, M. G. (2002). La enseñanza en el laboratorio: ¿qué podemos aprender en términos de conocimiento práctico y de actitudes hacia la ciencia? Enseñanza de las Ciencias, 20(3), 357-368.
Sias, D., Teixeira, R. (2006). Resfriamento de um corpo: a aquisição automática de dados propiciando discussões conceituais no laboratório didático de Física no ensino médio. Caderno Brasileiro de Ensino de Física, 23(3), 361-382.

Stake, R. E. (2010). Investigación con estudio de casos. Ediciones Morata.

Sureda, P., Otero, M. (2011). Nociones fundamentales de la teoría de los campos conceptuales. Revista electrónica de Investigación en Educación en Ciencias, 6(1), 124-138.

Torres, Á. (2010). Empleo del laboratorio asistido por ordenador en la enseñanza de la física y química de secundaria y bachillerato. Revista Eureka sobre Enseñanza y Divulgación de las Ciencias, 7(3), 693-707. https:// doi.org/10.25267/Rev Eureka ensen divulg cienc.2010.v7.i3.08

Vergnaud, G. (1990). La teoría de los campos conceptuales. Recherches en Didactique des Mathématiques, 10(2), 3.

Zohar, A. (2006). El pensamiento de orden superior en las clases de ciencias: objetivos, medios y resultados de investigación. Enseñanza de las Ciencias, 24(2), 157-172. 